\title{
Consumo de alcohol en escolares: descenso de la edad de inicio y cambios en los patrones de ingesta.
}

\author{
Espada Sánchez, J. P.; Méndez Carrillo, F. X.; Hidalgo Montesinos, M. D. \\ Departamento de Personalidad, Evaluación y Tratamiento psicológicos. Universidad de Murcia
}

Enviar correspondencia a:

JOSE PEDRO ESPADA SÁNCHEZ. Departamento de Personalidad, Evaluación y Tratamiento Psicológicos. Facultad de Psicología. Universidad de Murcia.

Campus de Espinardo. Apdo. 4021. 30080. Espinardo (MURCIA).E-mail: jpespada@fcu.um.es

\section{Resumen:}

Llevamos a cabo un estudio con el objetivo de describir la prevalencia del consumo de alcohol en una población de estudiantes de secundaria. Se aplicó una encuesta de 18 preguntas a una muestra representativa de 1013 sujetos (505 chicos y 508 chicas) seleccionados aleatoriamente por conglomerados, entre 10169 estudiantes de ESO en Elche (Alicante).

Se halló que el 67,7\% había consumido alcohol en alguna ocasión, de los que el 37,8\% eran bebedores habituales. La edad media de inicio en el consumo fue 12,2 años. La tasa de bebedores habituales se incrementa significativamente con la edad, pasando del $15,6 \%$ a los 12 años hasta el $83,1 \%$ a los 17 $(p=0,000)$. Las principales razones para iniciar el consumo son la curiosidad (42\%) y la presión social $(27,9 \%)$, observándose preferencia hacia las bebidas de alta graduación. Se describen también las circunstancias ambientales del consumo, así como las motivaciones hacia la bebida y la abstinencia. Se halló que a los 17 años es cuando mayor importancia se le da a la situación social como determinante de un eventual consumo. Las repercusiones para la salud $(39,1 \%)$ fue la principal razón manifestada en contra del abuso de alcohol.

Palabras clave: Alcohol, adolescentes, drogas legales, epidemiología

\section{Summary:}

The object of the following study is to describe the effects of the use of alcohol in secondary school students. An eighteen-question survey was made to a total of 1013 persons, 505 males and 508 females, that were randomly selected among 10169 students of ESO ("Enseñanza Secundaria Obligatoria", Compulsory Secondary School) in Elche, Alicante.

The survey showed that $67.7 \%$ of the interviewed students had taken alcohol at least a few times, and that $37.8 \%$ of them were habitual drinkers. The average age in which students usually start drinking, according to this survey, is when they are 12.2 years old. It is important to point out too the fact that the amount of drinkers increases proportionally with the age, changing from $15.6 \%$ when they are 12 , to $83.1 \%$ when they are $17 .(p=0.000)$. It seems to be proved that the main reasons to begin the use of alcohol are curiosity (42\%) and social pressure (27.9\%), and that youngsters are more prone to take beverages with a higher graduation of alcohol. In the study we also describe the environmental circumstances of the use of alcohol, as well as the different motivations for the use or not use of it. We came to prove that when they are seventeen, adolescents consider that the use of alcohol will improve their social situation and their relationships, so that this social situation can be presented as the main reason for an eventual use of alcohol. As a conclusion, we would like to point out that there are also voices raised against alcohol, whose main point is the undeniable fact that an abuse of alcohol can bring extremely negative consequences to human health. (39\%).

Key words: Alcohol, adolescents, legal drugs, epidemiology

\section{INTRODUCCIÓN}

E l abuso del alcohol supone un importante objeto de preocupación para la salud pública debido a sus repercusiones sociales y sanitarias. En la población general se asocia con problemas físicos: trastornos en el aparato digestivo, cánceres de esófago, hígado y páncreas (Tovar, 1994) y trastornos cardiovasculares (Casas, 1998). También conlleva una serie de secuelas psicológicas como demencia alcohólica (Caballería, Caballería y Parés, 1996), depresión
(De la Serna, 1996), suicidio (Nostrom, 1995; Revuelta y Kassem, 1996), celotipia (De la Serna, 1996; Secades, 1996), trastorno de pánico (Seguí et al., 1995), además de las considerables consecuencias sociofamiliares del alcoholismo, como la desestructuración familiar y problemática laboral.

Aunque muchas de las secuelas no aparecen a corto plazo, el organismo en desarrollo del adolescente se ve afectado de forma específica. El problema se acentúa cuando los consumidores son jóvenes: Hasta un $60 \%$ de los accidentes de tráfico se dan por la 
combinación de "jóvenes, alcohol y fin de semana" (Romero, 1994). El alcohol, además, constituye una droga de acceso al consumo de otras sustancias (Arévalo et al., 1997; Comas, 1994a). Otras repercusiones en población juvenil son las relaciones sexuales no planificadas, con riesgo de contraer el VIH o provocar embarazos no deseados (Cooper et al., 1994), los problemas legales como consecuencia de comportamientos agresivos y/o delictivos (Kachur et al., 1996; Otero et al., 1994; Vega, 1989) y bajo rendimiento académico (Perula et al., 1998; Vega, 1987).

Según el último informe del Plan Nacional sobre Drogas (1998) el consumo de alcohol es junto al tabaco y la heroína el que genera mayores problemas de salud en la sociedad española, lo que viene a confirmar que se precisan de acciones que contribuyan a comprender el fenómeno y a marcar líneas de intervención. Las actuaciones de carácter preventivo deben adaptarse a la realidad del problema en cada momento, como la edad en que se inicia el consumo. En el ámbito de la Comunidad Valenciana, un estudio desarrollado por la Dirección General de Drogodependencias de la Generalitat Valenciana en 1997 establecía en 16,6 años la edad en que se consume por primera vez, y una tasa de consumo de alcohol del $56,4 \%$ en población general.

En esta investigación nos hemos planteado los siguientes objetivos:

1. Conocer la prevalencia y tasas de consumo en escolares de las diferentes zonas escolares de la ciudad de Elche.

2. Estudiar variables mediadoras en el consumo de alcohol, como la ubicación del centro escolar, y diferencias en cuanto al sexo de los adolescentes.

3. Establecer la edad de inicio al consumo de bebidas alcohólicas.

4. Conocer los factores antecedentes y situacionales del consumo de alcohol entre adolescentes, en especial los determinantes del inicio al consumo de bebidas alcohólicas.

5. Describir y analizar las pautas actuales de consumo: cantidad, tipo de bebida, patrones de consumo de alcohol, etc.

6. Conocer las motivaciones de los adolescentes, tanto hacia el consumo, como hacia la abstinencia. 7. Conocer el grado de imitación por parte de los adolescentes de la conducta de beber de sus padres.

\section{MATERIAL Y MÉTODO}

\subsection{Sujetos}

La población objeto de estudio ha sido el conjunto de adolescentes escolarizados en los cursos pertenecientes a Educación Secundaria Obligatoria (y Bachille- rato antiguo) en el municipio de Elche (Alicante), localidad de 200.000 habitantes. Se han tomado en consideración los centros públicos y concertados que se ubican en el núcleo urbano del municipio.

Según datos de la Concejalía de Educación, el total de estudiantes de secundaria escolarizados en dichos cursos era 10.169 sujetos, agrupados en 359 aulas de 64 centros docentes.

Se realizó un muestreo por conglomerados, estableciendo el aula como unidad de selección, con un nivel de confianza de $95,5 \%$ y un margen de error de 0,03 .

Establecimos un número proporcional de aulas por cada una de las zonas escolares, de manera que las diferentes zonas de la ciudad estuvieran representadas en función de su población, y se seleccionaron aleatoriamente 33 aulas que conformaron la muestra, con un total de 1013 sujetos pertenecientes a 25 centros escolares.

Por sexos, la muestra se distribuye de manera equitativa: $49,8 \%$ varones y $50,2 \%$ mujeres. Respecto a la edad, el grueso de la muestra se concentra entre los 12 y los 15 años (87,26\%). La distribución de frecuencias en función del sexo y la edad se presenta en la Tabla 1.

\section{Tabla 1.- Distribución de los sujetos de la muestra en función del sexo y edad.}

\begin{tabular}{ccc}
\hline Sexo & $\mathbf{N}$ & $\mathbf{\%}$ \\
\hline Varón & 505 & $49,85 \%$ \\
Mujer & 508 & $50,15 \%$ \\
\hline Edad & $\mathbf{N}$ & $\%$ \\
\hline 11 & 7 & $0,69 \%$ \\
12 & 281 & $27,74 \%$ \\
13 & 275 & $27,15 \%$ \\
14 & 159 & $15,69 \%$ \\
15 & 169 & $16,68 \%$ \\
16 & 89 & $8,78 \%$ \\
17 & 33 & $3,26 \%$ \\
\hline TOTAL & 1013 & $100,00 \%$ \\
\hline
\end{tabular}

\subsection{Instrumentos de evaluación}

Para la recogida de información se utilizó el CUESTIONARIO DE HÁBITOS Y MOTIVACIONES AL CONSUMO DE ALCOHOL (CHC), elaborado para este estudio. Se trata de un cuestionario compuesto por 18 ítems, y que consta de tres partes: Instrucciones, datos de identificación (el cuestionario es anónimo, se pedía la edad, sexo y centro de estudio) y preguntas agrupadas en dos apartados: "La primera vez que bebiste" y "Si bebes actualmente." Los ítems eran de opción múltiple entre 2-7 alternativas. En el cuestiona- 
rio se incluyeron cuatro ítems adaptados de la Escala de Implicación con el Alcohol para Adolescentes (AAIS), de Mayer y Filstead (1979). Previamente a su utilización se realizó un estudio piloto para depurar el instrumento.

\subsection{Procedimiento}

La aplicación del cuestionario se realizó durante los meses de mayo y junio de 1998, en horario escolar, de forma colectiva, en las aulas de los centros seleccionados. Previamente se informó a la dirección del centro de los objetivos y procedimiento de la investigación. Un investigador explicó a los alumnos/as la forma de responder el cuestionario, garantizando el anonimato, y comprobó la cumplimentación de todos los ítems, aclarando aspectos que precisaran explicación.

En una segunda fase, y una vez reunidos todos los cuestionarios aplicados, las respuestas fueron introducidas en una base de datos, y posteriormente procesadas mediante el paquete informático SYSTAT v.5.01 (Wilkinson, 1990)

\section{RESULTADOS}

\subsection{Consumo de alcohol}

El $67,7 \%$ de los adolescentes encuestados manifiesta haber probado en alguna ocasión una bebida alcohólica, de los que el 37,8\% beben actualmente con cierta frecuencia y el 29,9\% lo probó pero en el presente no bebe. El 32,3\% restante nunca ha bebido (Figura 1).

Entre los sujetos que habían tomado alcohol alguna vez, encontramos que el 6,5\% había bebido hacía menos de una semana, el $42 \%$ hacía menos de un mes, un 33,8\% hacía menos de un año, y un 16,9\% hacía más de un año.

Conforme aumenta la edad, la tasa de bebedores habituales es significativamente más alta $\left(\mathrm{G}^{2}=257,866\right.$, $p=0,000)$. A los doce años es de 15,6\%, alcanzando el $78,7 \%$ a los 17 . El máximo porcentaje se encontró a los 16 años $(83,5 \%)$



Figura 1: Consumo de alcohol entre los escolares de la muestra
El porcentaje de varones que han bebido alcohol en alguna ocasión es 70,1\%, frente al 65,3\% de mujeres. La diferencia, pese a ser de cinco puntos no resulta significativa, y no es posible afirmar que la variable "sexo" predispone o condiciona el eventual consumo de alcohol. $\left(\mathrm{G}^{2}=2,61, \mathrm{p}=0,106\right)$.

La media de edad de inicio al consumo en la muestra estudiada es de 12,2 años, con una desviación típica de 1,67. En la Figura 2 se muestra el número de adolescentes que comienzan a beber en cada edad.

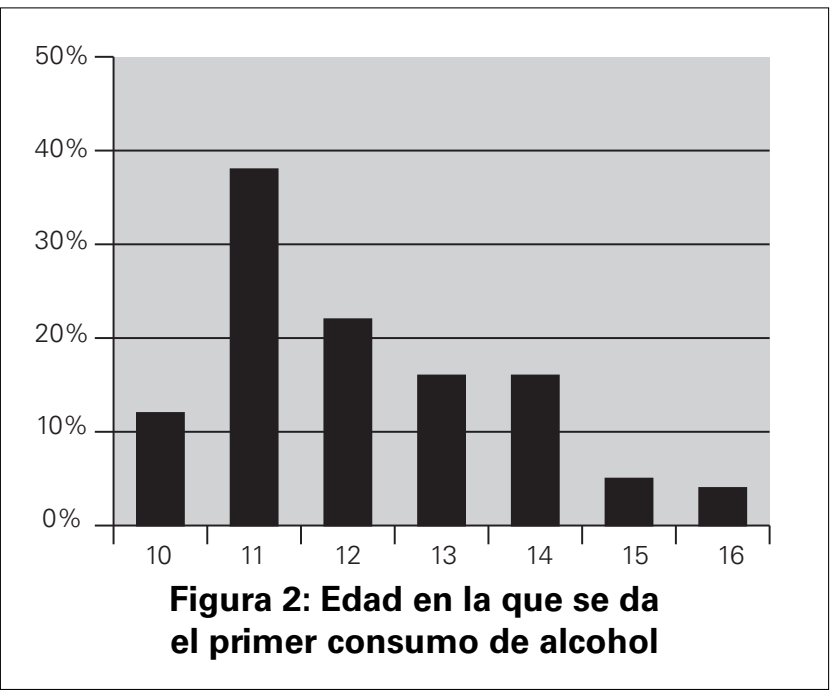

Observando la relación entre consumo de alcohol y la zona escolar donde se sitúa cada centro educativo, encontramos que existe una mayor tasa de consumidores en la zona Sureste (76\%) y Noroeste $(70,9 \%)$

Podría afirmarse que existe una relación entre la variable "zona escolar" y consumo de alcohol. No obstante, no hemos tomado este dato como determinante, debido a la desigual distribución de centros educativos en las zonas escolares de la ciudad: En las zonas Sureste y Noroeste se ubica un mayor número de aulas pertenecientes a Institutos de Secundaria, donde se encuentran escolarizados estudiantes de mayor edad. En la zona Centro, sin embargo, no existe ningún Instituto de Educación Secundaria.

\subsection{Motivos para el primer consumo}

Nos referiremos en este y en el siguiente apartado a los adolescentes que han bebido en alguna ocasión. La curiosidad es la razón principal a la hora de probar por primera vez una bebida alcohólica (42\%). Le sigue la presión social ("porque me invitaron o animaron") $(27,9 \%)$, lo que muestra la vinculación del alcohol a situaciones sociales y la búsqueda de efectos fisiológicos ("para colocarme") (7,3\%). En el apartado de "otras razones" los encuestados dan respuestas como "por probarlo", y señalan el papel del contexto en que se encontraban como motivo para consumir: "para celebrar algo" o "porque era Navidad". 


\subsection{Circunstancias del consumo actual}

Prácticamente la totalidad de los escolares encuestados que beben actualmente lo hacen uno $(72,4 \%)$ o dos $(20,5 \%)$ días a la semana.

Los adolescentes de la muestra beben alcohol predominantemente solo durante los fines de semana $(87,7 \%)$ El $7 \%$ lo hace durante toda la semana, y el $5,3 \%$ únicamente entre semana. Las bebidas más consumidas son los licores $(32,9 \%)$ y los combinados $(32,5 \%)$ En menor medida la cerveza $(15 \%)$ y el vino $(12,7 \%)$. Los principales lugares de consumo los bares, pubs y discotecas $(58 \%)$ y el propio domicilio $(21,1 \%)$.

Entre los momentos del día en que los adolescentes consumen bebidas alcohólicas, es la noche el preferido $(76 \%)$, aunque un $22,6 \%$ de los sujetos informaron que solían beber por la tarde y un porcentaje mínimo lo hacía durante la mañana $(1,4 \%)$.

En cuanto a los acompañantes mientras se bebe alcohol, más de la mitad de los escolares encuestados consumen con amigos de su misma edad. Un tercio de la muestra dicen beber únicamente en presencia de sus padres y otros familiares (Figura 3).

Los adolescentes que beben en la actualidad manifiestan que el principal motivo para mantener el consumo es la posibilidad de pasárselo mejor $(44,3 \%)$, y en menor medida lo hacen para desinhibirse $(5,7 \%)$ y "para ligar más" (3,5\%). En este ítem, se dio una alta tasa de respuesta de los encuestados hacia la respuesta "otros motivos": En este apartado, entre otros señalan "porque me gusta", o "porque tengo sed", "para celebrar algo" y "por probar una bebida".
(10\%) manifestaban haber alcanzado la intoxicación etílica en alguna ocasión.

Los sujetos de mayor edad refieren haber experimentado más efectos como consecuencia de la bebida $\left(r_{x y}=0,444\right)$. Entre varones y mujeres no se aprecian diferencias estadísticamente significativas. No obstante, en los ítems referentes a los efectos más severos, como "sentirse enfermo", "desmayarse", las chicas obtuvieron porcentajes de hasta tres puntos de diferencia.

\subsection{Expectativas respecto al consumo}

Hemos preguntado a los adolescentes sobre su intención de beber en un futuro, tanto si ya bebían en la actualidad, como si no. Existen diferencias significativas entre sexos, al observar las expectativas de futuro respecto al consumo de alcohol. $\left(\mathrm{G}^{2}=22,036\right.$, $p<0.000)$. En este sentido, parece que los varones esperan a tener más edad para beber. Las mujeres, por su parte, manifiestan una menor intención de beber pero tomarían su decisión en función del momento concreto en que surgiera beber.

En función de que los sujetos hayan probado o no el alcohol, también se dan diferencias significativas $(\mathrm{G} 2=83,969, \mathrm{p}<0.000)$. Entre los que han bebido, un gran porcentaje bebería en función de la situación concreta en que se planteara la posibilidad de beber. Entre los que no han bebido nunca, la mitad supeditan su decisión al contexto.

Por edades también se observan distintas tendencias en la intención sobre futuros consumos de alcohol (Figura 4). La respuesta "No tengo intención de

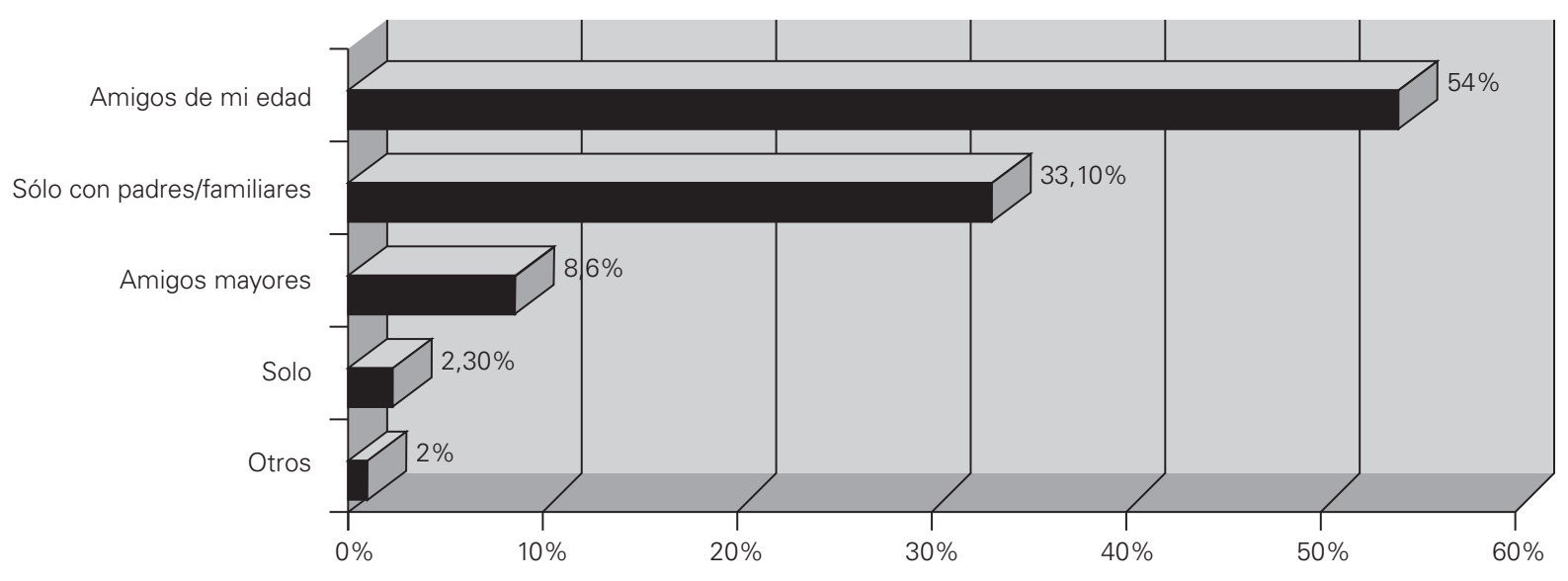

Figura 3: Compañeros de bebida

Respecto a los efectos alcanzados a través de la bebida, la mayoría de los sujetos que han bebido dicen no haber experimentado ningún efecto provocado por el alcohol (58\%), Algunos estados alcanzados fueron desinhibición $(7,1 \%)$ y sentirse enfermo y/o desmayarse $(8,6 \%)$. Un total de 108 adolescentes beber" se da mayoritariamente a los 12 años (52,4\%), descendiendo conforme se avanza en edad, hasta el $30,3 \%$ a los 17 años. A los 16 y 17 años es cuando mayor importancia se da al contexto en el que podría presentarse el consumo. La mayoría contesta que el beber o no "depende de la situación". 
Se ha estudiado también los motivos para no consumir alcohol en la actualidad entre los chicos/as que sujetos que han señalado positivamente esa respuesta. La razón que más adolescentes señalan es la preo-

Tabla 2: Expectativas de consumo

\begin{tabular}{|c|c|c|c|c|c|c|c|c|}
\hline & \multicolumn{2}{|c|}{ Varones } & \multicolumn{2}{|c|}{ Mujeres } & \multicolumn{2}{|c|}{ Consumidores } & \multicolumn{2}{|c|}{ Abstemios } \\
\hline & $\mathrm{N}$ & $\%$ & N & $\%$ & $\mathrm{~N}$ & $\%$ & $\mathrm{~N}$ & $\%$ \\
\hline No tengo intención de beber & 229 & 22,6 & 250 & 24,7 & 262 & 38,1 & 217 & 66,3 \\
\hline Depende de la situación & 157 & 15,5 & 192 & 18,9 & 280 & 40,1 & 69 & 21,1 \\
\hline Beberé cuando tenga más edad & 76 & 7,5 & 49 & 4,8 & 89 & 12,8 & 36 & 11,0 \\
\hline Creo que empezaré pronto a beber & 16 & 1,6 & 7 & 0,7 & 20 & 2,9 & 3 & 0,9 \\
\hline NS/NC & 27 & 2,7 & 10 & 1,0 & 35 & 5,1 & 2 & 0,6 \\
\hline TOTAL & 505 & 100,0 & 508 & 100,0 & 686 & 100,0 & 327 & 100,0 \\
\hline
\end{tabular}

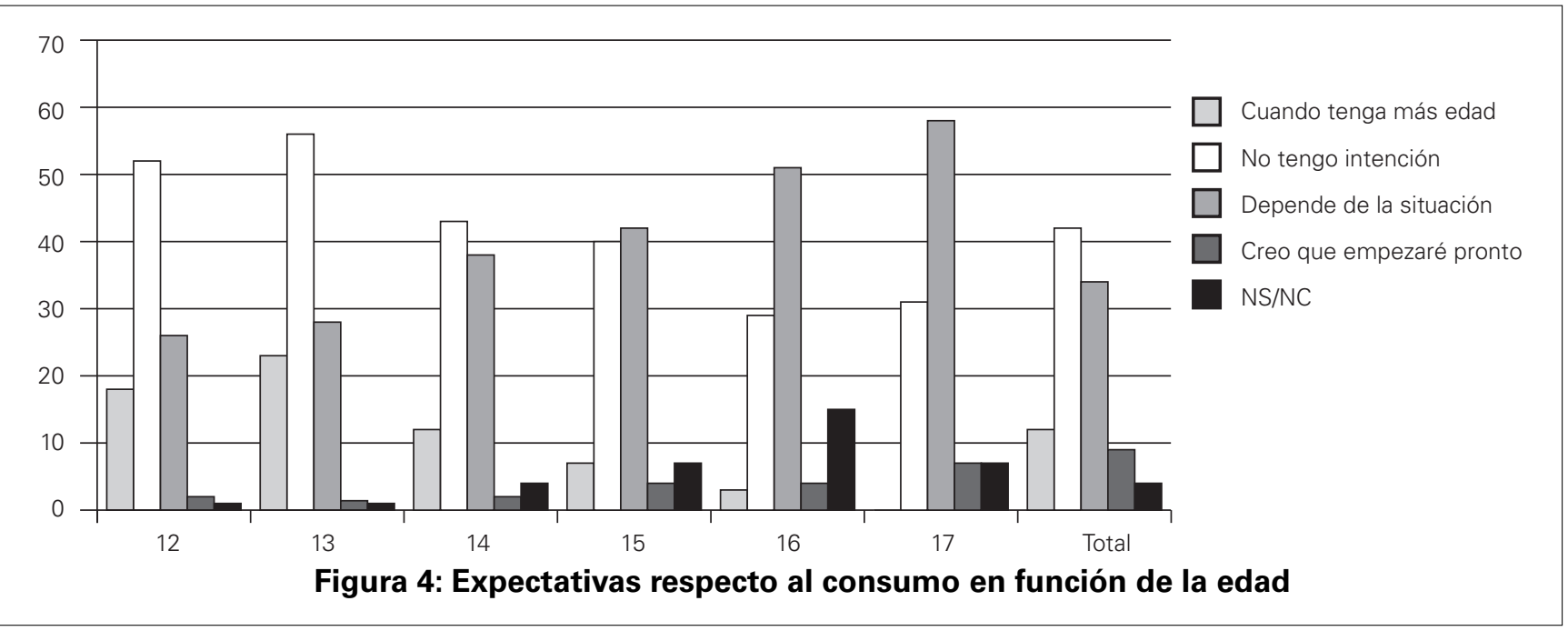

no han bebido nunca, o que ha pasado más de un mes desde la última vez que tomaron alcohol (que suponen 483 sujetos). A continuación se describe separadamente cada una de las razones aducidas, de forma que en la Figura 5 se muestra el porcentaje de cupación por las consecuencias en la salud derivadas de tomar alcohol, seguido por "no querer perder el control" y "no me gustan las bebidas con alcohol."

En general no existen diferencias estadísticamente significativas entre varones y mujeres en cuanto a las

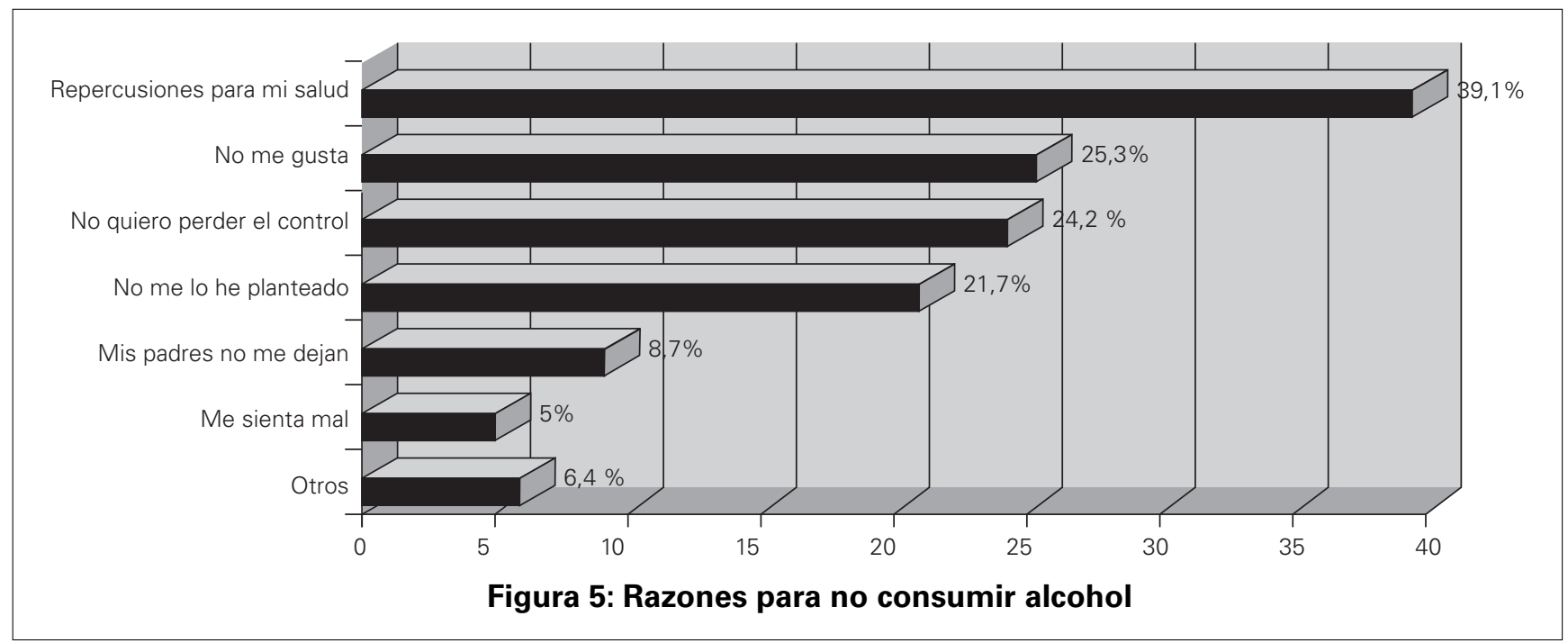


razones para no consumir, la única respuesta en la que se encontraron diferencias en función del género fue "temor por perder el control del comportamiento" $\left(G^{2}=11,518, p=0.003\right)$. En este caso hubo un mayor porcentaje de chicas que dieron esta razón frente al porcentaje de hombres.

\subsection{Imitación familiar}

Hemos estudiado la relación entre la variable "consumo de alcohol por parte de los padres" y el consumo de los hijos. Hallamos que no existían diferencias estadísticamente significativas entre los padres de los chicos/as que han probado el alcohol y los que no lo han hecho. Sin embargo, se puede apreciar que los padres con hijos bebedores acostumbran a beber a diario un $5 \%$ más que los padres de hijos que no beben. Entre los adolescentes que beben, un 17,2\% de los padres beben alcohol casi a diario, mientras que en el caso de los menores que no bebe, el porcentaje de padres bebedores a diario se reduce al $12,8 \%$. (Figura 6)

\section{DISCUSIÓNY CONCLUSIONES}

Una encuesta realizada entre escolares de todo el territorio español por el Plan Nacional sobre Drogas (1997), encontraba una tasa de bebedores del 66\%. Desde un ámbito autonómico (Generalitat Valenciana 1997), se halló una prevalencia del 54\%. En nuestra investigación encontramos que el $67,7 \%$ de escolares habían bebido en alguna ocasión. Esta tasa es mayor que la encontrada en la Comunidad Valenciana, y muy similar a la del resto del territorio español.

La edad de inicio hallada en nuestra muestra resulta notablemente más baja que la obtenida en el citado estudio de la Generalitat Valenciana, la edad global media de inicio era 16,16 años que realizaba una exploración retrospectiva del inicio al consumo, estando la información influida por la memoria de los encuestados. Jiménez-Bueno et al. (1997) hallaron que en el área de Alicante el 38,1\% se iniciaban en el consumo antes de los 14 años. En nuestra muestra, de edades mas tempranas observamos mayor preco-

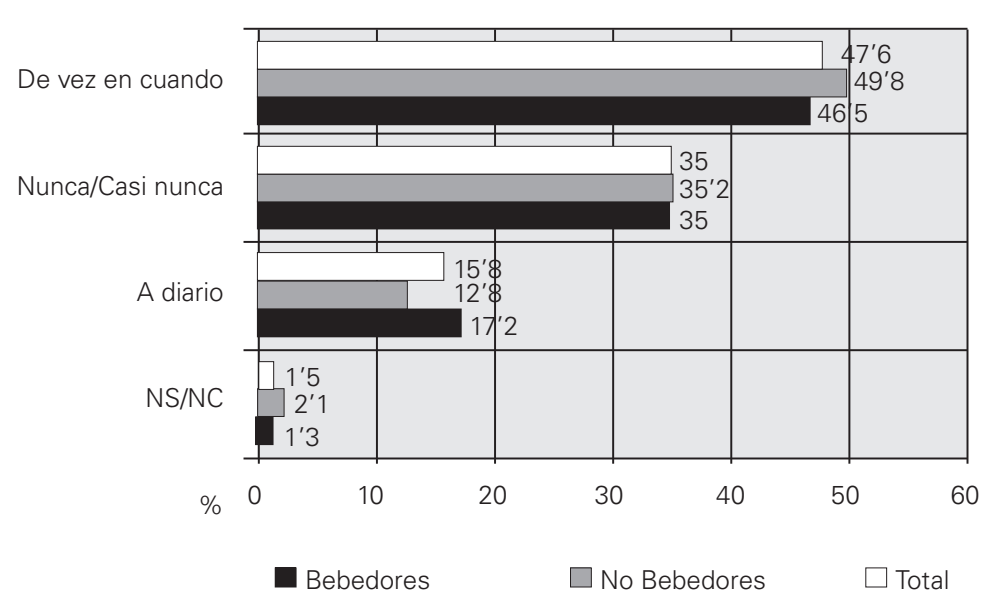

Figura 6: Consumo por parte de los padres

cidad en el inicio a la ingesta de bebidas alcohólicas (12,2 años). Se detecta, a pesar de las diferencias en la metodología de los estudios, que actualmente los adolescentes comienzan antes a beber.

Los varones no comienzan el consumo de alcohol a una edad más temprana que las mujeres: Las edades halladas han resultado casi idénticas para ambos sexos (12,1 en chicos y 12,2 en chicas). Otros estudios encontraron diferencias aproximadamente de medio año de "adelanto" por parte de los varones (Generalitat Valenciana,1997) o incluso de dos años, (García Jiménez et al. 1993). Por sexos se observa un cambio en la tendencia observada en estudios previos (Cárdenas, 1986; Comas, 1994b; Ministerio de Sanidad y Consumo, 1994), que señalaban una mayor precocidad en el caso de los varones. Algunos estudios apuntaban ya una reducción de las diferencias de con- sumo entre sexos (Basabe y Páez, 1992; Villalbí et al., 1995), llegando incluso a superar el consumo de las mujeres al de los varones (Casado et al., 1998).

Otra variable en relación con el sexo es la cantidad de varones bebedores respecto de las mujeres. En este sentido, no se han hallado diferencias significativas en cuanto al consumo en función del sexo de los adolescentes.

Acerca de los efectos que alcanzaron mediante el consumo de alcohol, las chicas obtuvieron porcentajes algo mayores. Esta disparidad podría deberse a una menor tolerancia al alcohol, influida por el menor peso corporal y el funcionamiento metabólico femenino.

Las variables que acompañan y/o desencadenan el primer consumo de alcohol ayudan a entender mejor el fenómeno del consumo abusivo de alcohol. Si bien todo consumo -más o menos esporádico- no conlleva 
una adicción o un abuso, sí puede mostrar los mecanismos por los que se rige el consumo de alcohol y otras drogas: encontrarse en una situación social, con una motivación determinada, la curiosidad, etc.

En el caso concreto del alcohol, por tratarse de una droga legal, y que cuenta con una presencia muy arraigada socialmente, el primer consumo frecuentemente se da con la permisividad paterna, a diferencia de otras sustancias.

En cuanto a los motivos por los que los adolescentes no consumen alcohol, se observa una concienciación alta acerca de las repercusiones del alcohol en la salud, siendo éste el principal motivo al que los estudiantes han aludido como razón para no beber alcohol.

El patrón de consumo que se observa es de fines de semana, por la noche, en bares, pubs y discotecas y en compañía de amigos de su edad y/o más mayores. Tal y como apuntaba Rooney (1990) uno de los cambios del consumo de alcohol en la cultura española es precisamente que se cambia el lugar de consumo del hogar por los bares.

Las bebidas más consumidas son licores y combinados. Se observa un descenso en el consumo de otras bebidas como la cerveza o la sidra, que eran las preferidas según otros estudios realizados con población escolar (Alonso et al., 1997; García Jiménez et al. 1993; Robledo et al., 1996). Esta diferencia en la elección de bebida podría tener dos explicaciones: un cambio en las preferencias por parte de los consumidores o también la opción por bebidas de alto contenido alcohólico por sus mayores efectos embriagantes. Siguiendo a Marina (1998), "no es tanto beber como haber bebido lo que incita a consumos de alcohol de alta graduación".

Parece que una motivación importante es el deseo de buscar sensaciones, concretamente la euforia y embriaguez. Podemos destacar que en la muestra estudiada, un porcentaje significativo afirmaba haberse "colocado" bebiendo alcohol, o haberse emborrachado en alguna ocasión. Este dato viene a reforzar la idea de que el consumo de alcohol está pasando de tener una función social, o de disfrute de la bebida, a la de provocar estados de evasión.

Las situaciones de interacción social, en especial entre el grupo de iguales son en las que se dan la mayoría de consumos de alcohol por parte de los adolescentes. En esta línea han concluido otras investigaciones, como las realizadas por Ariza y Nebot (1995) o Basabe y Páez (1992).

El alcohol, por tanto, y desde el punto de vista adolescente, parece tener una función "socializadora", que facilita la interacción con otras personas, y sobre todo supone una fuente o vía de diversión.

En estos momentos los adolescentes y jóvenes tienen muy asociados la diversión y el ocio, especialmente de fin de semana, con el consumo de sustan- cias psicoactivas, por lo que, como hemos indicado en otros lugares se hacen necesarias estrategias dirigidas a promover un uso saludable del tiempo libre (Espada, Sarabia y Lillo, 1998; Macià, Olivares y Méndez, 1990).

Este estudio ha sido financiado con una Ayuda a la Investigación del Instituto de Cultura Juan Gil-Albert, de la Diputación Provincial de Alicante

\section{REFERENCIAS BIBLIOGRÁFICAS}

Alonso, J., Rosado. J., Ruiz, R. y Alonso, J. (1997): Consumo de alcohol y adolescencia: Estudio epidemiológico descriptivo. Atención primaria, 19, 4, 183-187

Caballería, LI, Caballería, J.; y Parés, A.(1996): Consulta diaria. Qué haría usted ante un paciente con alcoholismo crónico. Medicina integral, 27, 4, 125-132.

Arévalo, J. M.; Masip, G.P. y Abecia, L.C. (1997): Consumo de alcohol en una muestra de estudiantes universitarios. Revista Española de Drogodependencias, 22, 1, 15-34.

Ariza, C. y Nebor, M. (1995): Consumo de bebidas alcohólicas en la adolescencia. Atención primaria (II), 1,26-31.

Basabe, N. y Páez, D. (1992): Los jóvenes y el consumo de alcohol. Representaciones sociales. Bilbao: Fundamentos

Casado, C., González, S., Martínez, N. y López, I. L. (1998): Consumo de tabaco, alcohol y cannabis en estudianes de secundaria de Oviedo. Enfermería científica, 194$195,10-14$.

Cárdenas, C. (1986): Consumo de bebidas alcohólicas en la adolescencia. Condiciones contribuyentes. Drogalcohol, 11, 2, 58-65.

Casas, J. (1998): Actitudes, habilidades sociales y autocontrol en la prevención del consumo abusivo de alcohol y tabaco. Tesis Doctoral. Madrid: Universidad Autónoma.

Comas, D. (1994a): Alcohol y adolescencia: claves de interpretación. En alcohol y adolescencia: experiencia y programas de educación preventiva: actas del II Congreso de prevención desde la comunidad educativa. Madrid: FERE, 31-46.

Comas, D. (1994b): Los jóvenes y el uso de drogas en la España de los anos 90. Madrid: Ministerio de Asuntos Sociales. Instituto de la Juventud.

Cooper, A. L.; Richter, D. L.; Valois, R. F.; Mac Keown, R. E.; Garrison, C. Z. y Vincent, L. O. (1994): Correlates and consequences of early initiation of sexual intercouse. Journal of School health, 64, 9, 372-377.

De la Serna, I. (1996): Complicaciones psiquiátricas del alcoholismo. En A. Valbuena y C. Álamo (Coords.): Avances en toxicomanías y alcoholismo.

Espada, J.P., Sarabia, F. y Lillo, N. (1998): Los Centros Juveniles como recurso para la prevención de la drogodependencia. Congreso de alcohol, drogas de síntesis y 
alternativas de ocio y tiempo libre para jóvenes. Madrid: Cruz Roja.

García Jiménez , M.T.; Gamonal, A.; Amela, C.; Casas, J.; Dorado, L.; Herce, P.; Ibáñez, C.; López, C.; Mazarrasa, L.; Marco, S.; Munugarren, R.; Nájera, P. y Pachón, I.(1993): Estudio sobre el consumo juvenil de bebidas alcohólicas en la comunidad de Madrid. Madrid: Consejería de Integración social e Instituto de Salud Carlos III.

Generalitat Valenciana (1997): El consumo de drogas y factores asociados en la Comunidad Valenciana. Dirección General de Drogodependencias. Conselleria de Benestar Social.

Jiménez-Bueno, M.C.; Cabrero, E.; Porres, D.; Luna, J. D. y Luna, A. (1997): El consumo de bebidas alcohólicas durante el fin de semana por adolescentes en el área de Alicante. Adicciones, 9,2,171-179.

Kachur, S.P.; Stennies, G.M.; Powell, K. E.; Modzeleski, W.; Stephens, R.; Murphy, R.; Resnox, M.,

Sleeto, D. y Lowry, R. (1996): School associated violent deaths in the United States. 1992-1994. Journal American Medical Asscociation, 274,22,1729-1733.

Macià, D. (1995): Las drogas: conocer y educar para prevenir. Madrid: Pirámide.

Macià, D.; Olivares, J. y Méndez, F.X. (1990): Alternativas de tiempo libre en la prevención de las drogodependencias, VIII Congreso Nacional de Psicología, Barcelona.

Marina, J. A. (1998): El fenómeno social y el consumo de alcohol y drogs de síntesis entre jóvenes. Congreso de Alcohol, drogas de Síntesis y alternativas de ocio y tiempo libre para jóvenes.

Mayer, J. y Filstead, W. J. (1979): The adolescent alcohol involvement Scale. An instrument for measuring adolescents' use and misure of alcohol. Journal of Studies on alcohol, 3, 291-300.

Ministerio de Sanidad y Consumo (1994): Alcohol y Salud Pública. Secretaría General de Salud. Dirección General de Salud Pública.

Nostrom, T. (1995): Alcohol and suicide: a comparative analysis of France and Sweden. Addiction, 90, 1, 1463-1469.

Otero, J.M.; romero, E. y Luengo, A. (1994): Identificación de factores de riesgo de la conducta delictiva: Hacia un modelo integrador. Análisis y modificación de conducta, 20,73,675-709.
Perula, L. A.; Ruiz, R., Fernández, J.A.; Herrera, E., De Miguel. M. D. y Bueno, J. M. (1998): Consumo de alcohol entre los escolares de una zona básica de salud de Córdoba. Revista Española de Salud Pública. 72, 4, 331341.

Plan Nacional Sobre Drogas (1997): Encuesta sobre drogas a la población escolar: 1996. Madrid: Ministerio de Interior.

Plan Nacional Sobre Drogas (1998): Memoria 1997. Madrid: Ministerio de Interior.

Revuelta, I. y Kassem, S. (1996): Los trastornos depresivos en atención primaria: Epidemiología, clasificación y diagnóstico. Medifam, 6, 3, 150-157.

Robledo, T.; Rubio, J. y Espiga, I. (1996): Consumo de alcohol en jóvenes: un reto para nuestra sociedad. En E. Gil, T. Robledo, J. Rubio e I. Espiga: alcohol y Juventud 1995: Madrid: Ministerio de Sanidad y Consumo.

Romero, J. M. (1994): Alcoholismo Juvenil. Entorn Actual, 21, 13-17.

Rooney, J. F. (1990): Funciones sociales de los bares para la juventud en España. Adicciones, 2, 3, 209-220.

Secades, R. (1996): Alcoholismo Juvenil: Prevención y tratamiento. Madrid: Pirámide.

Seguí, J.; Salvador, L.; Canet, J.; Aragón, C. y Herrera, C. (1995): Comorbilidad entre el trastorno de pánico y alcoholismo (II): alcoholismo en una muestra de 148 pacientes con trastornos de pánico. Actas Luso-Españolas de Neurología, Psiquiatría y Ciencias afines, 23, 6, 288-292.

Tovar, C. (1994): Alcoholismo. En C. Eseverri; J. A. Diego, A. Terán, C. Tovar; J. A. Galán, A. I. Arredondo y C. Couceiro: Educar para la salud: Drogodependencias. Madrid: CCS.

Vega, A. (1987): El consumo de alcohol en el País Vasco: Problemática y respuestas. Revista Española de Drogodependencias, 1, 35-40.

Vega, (1989): Menores, delincuencia y drogas. Revista Española de Drogodependencias, 14,4,244-285.

Villalbí, J.R.; Nebor, M. y Ballestín, M.(1995): Los adolescentes ante las sustancias adictivas: tabaco, alcohol y drogas no institucionalizadas. Medicina Clínica, 104, 784-788.

Wilkinson, L. (1990): SYSTAT: The system for Statistics. Evanston, IL: SYSTAT Inc. 